\title{
Acute polymyositis in an adult associated with Mycoplasma pneumoniae infection
}

\author{
M.R. Bennett, H.J. O'Connor, J.M. Neuberger D.M. and E. Elias \\ Department of Gastroenterology, Queen Elizabeth Hospital, Birmingham B15 2TH, UK.
}

\begin{abstract}
Summary: A previously healthy adult developed a hitherto unreported major complication of Mycoplasma pneumoniae infection, namely acute polymyositis.
\end{abstract}

\section{Introduction}

Infection with Mycoplasma pneumoniae may be associated with a variety of extrapulmonary manifestations. ${ }^{\prime}$ Cardiovascular manifestations such as myocarditis and pericarditis, and neurological complications such as meningitis and cranial nerve palsies are well described, together with a variety of skin rashes and haematological abnormalities. Musculoskeletal involvement is usually limited to a mild transient myalgia or arthralgia occurring in $15-45 \%$ of patients. ${ }^{2,3} \mathrm{We}$ describe a hitherto unreported major complication of $M$. pneumoniae infection in an adult, namely polymyositis.

\section{Case report}

A 23 year old previously healthy man was admitted with a 10 day history of progressive generalized weakness, pyrexia and a cough productive of mucopurulent sputum. He had taken oral amoxycillin for the 5 days prior to admission. On examination the patient was unable to stand unaided and there was clinical evidence of generalized muscle weakness, most marked in the proximal limb muscles, with associated swelling and tenderness. There was no skin rash or sensory disturbance and limb and plantar reflexes were normal. Coarse crepitations were audible at the base of the left lung.

Investigations on admission showed a haemoglobin of $17.2 \mathrm{~g} / \mathrm{dl}$, white cell count $15.7 \times 10^{9} / 1$ with a neutrophil leucocytosis. and an ESR of $5 \mathrm{~mm} / \mathrm{h}$.

Correspondence: M.R. Bennett M.R.C.P., Department of Cardiology, General Hospital, Birmingham B4 6NH, UK.

Accepted: 26 May 1989
Serum urea, creatinine and electrolytes were normal. Serum aspartate aminotransferase was elevated at $820 \mathrm{U} / 1$ (normal range up to $35 \mathrm{U} / \mathrm{l}$ ) and his serum creatine kinase was $>100,000 \mathrm{U} / 1$ on two separate estimations (normal range up to $80 \mathrm{U} / 1)$, with normal cardiac isoenzymes. Urinalysis showed proteinuria but no haematuria or myoglobinuria and a 24-hour urinary protein was elevated at $1.9 \mathrm{~g} / \mathrm{l}$ (normal range up to $0.3 \mathrm{~g} / \mathrm{l}$ ). Peak expiratory flow rate was reduced at 350 litres/min although his chest X-ray was normal. Electromyography showed a myopathic picture and a muscle biopsy on the second hospital day showed features consistent with a low-grade myositis. Antinuclear factor, antismooth muscle and antimitochondrial antibodies by immunofluorescent assay were negative; immune complexes were not detected in the blood; serum levels of IgG, $\mathrm{A}$ and $\mathrm{M}$ and of $\mathrm{C} 3$ and $\mathrm{C} 4$ complement were normal and there was no detectable abnormality of lymphocyte surface antigens. Serological tests for Coxsackie virus, influenza virus $A$ and $B$, adenovirus, echovirus, mumps virus, respiratory syncytial virus, Legionella, toxoplasmosis, Coxiella burneti, Chlamydia and Trichinella were negative. However, the complement fixing antibody titre to $M$. pneumoniae was 1:256 on admission rising to 1:512 seven days later.

The patient was treated with bed rest and physiotherapy and showed a marked improvement in both muscle power and general well-being by the sixth hospital day. Peak expiratory flow rate rose to normal levels coincident with a rapid fall in serum creatine kinase. The 24-hour urinary protein fell to $0.6 \mathrm{~g} / \mathrm{l}$. The patient was discharged fully ambulant on the tenth hospital day. The titre of antibody to $M$. pneumoniae had fallen to $1: 32$ at 3 months after the illness and the patient remains well at 18 months of follow-up. 


\section{Discussion}

The diagnosis of infection with $M$. pneumoniae rests on the association between suggestive clinical features and appropriate serological evidence. In our patient, a pneumonic illness was accompanied by a greater than four-fold fall in specific antibody titre to $\boldsymbol{M}$. pneumoniae between the acute and convalescent sera. The organism may be isolated from throat swabs and sputum but only rarely from blood ${ }^{4}$ or cerebrospinal fluid, ${ }^{5}$ even when extrapulmonary manifestations are present. Cold agglutinins are found in the serum in about $50 \%$ of cases, but are non-specific. ${ }^{1}$ Infection with $M$. pneumoniae generally runs a benign course. Extrapulmonary manifestations usually occur within 2 weeks of the onset of the respiratory illness and are not affected by treatment of the underlying pneumonia.

The pathogenesis of the extrapulmonary manifestations of $M$. pneumoniae infection is unclear. Occasionally, M. pneumoniae may be isolated from the tissue sites involved ${ }^{5}$ suggesting direct invasion, and indeed in myositis associated with Coxsackie virus infection or toxoplasmosis, the

\section{References}

I. Murray, H.W., Masur, B., Senterfit, L.B. \& Roberts, R.B. The protean manifestations of Mycoplasma pneumoniae infections in adults. Am J Med 1975, 58: 229-242.

2. Feizi, T., Maclean, H., Somerville, R. \& Selwyn, J.G. Studies on an epidemic of respiratory disease caused by Mycoplasma pneumoniae. Br Med J 1967, i: 457-460.

3. Mufsen, M.A., Manko, M.A., Kingston, J.R. \& Chanock, R.M. Eaton agent pneumonia-clinical features. JAMA 1961, 178: $369-374$.

4. Naftalin, J.M., Wellisch, G., Kahana, Z. \& Diengott, D. Mycoplasma pneumoniae septicaemia. JAMA 1974, 228: 565 .

5. Bayer, A.S., Galpin, J.E., Theofilopoulos, A.N. \& Guze, L.B. Neurologic disease associated with Mycoplasma pneumoniae pneumonitis. Demonstration of viable Mycoplasma pneumoniae in cerebrospinal fluid and blood by radioisotopic and immunofluorescent tissue culture techniques. Ann Int Med 1981, 94: 15-20.

6. Mastaglia, F.L. \& Walton, J.N. Coxsackie virus-like particles in skeletal muscle from a case of polymyositis. J Neurol Sci 1970, 11: 593-599. organisms have been demonstrated in skeletal muscle. ${ }^{6.7}$ However, it is thought that $M$. pneumoniae exerts its cytopathic effect while adhering to the outer surface of epithelial membranes and intracellular invasion has not been demonstrated in experimental infection. ${ }^{8}$ No evidence has been found for toxin production by $M$. pneumoniae. Although autoantibodies were not found in our patient, a variety of antibodies have been demonstrated in infection with $M$. pneumoniae which crossreact with human heart, lung, liver and brain, and circulating immune complexes may be detected in the acute phase of the illness. ${ }^{10}$ Finally, infection with $M$. pneumoniae may be associated with a hypercoagulable state, ${ }^{11}$ and intravascular coagulopathy has been suggested as a pathogenetic mechanism to account for distant complications, although in this case, the patient had a normal blood clotting profile and undetectable levels of serum cold agglutinins.

In conclusion, although there is good evidence to implicate Mycoplasma pneumoniae as the aetiological agent in this patient's polymyositis, the exact mechanism by which it may produce muscle damage still remains unclear.

7. Callahan, Jr., W.P., Russell, W.O. \& Smith, M.G. Human toxoplasmosis. Medicine 1946, 25: 353-397.

8. Fernald, G.W. \& Clyde, Jr, W.A. Pulmonary immune mechanisms in Mycoplasma pneumoniae disease. In: Kirkpatrick, C.H. and Reynolds H.Y. (eds) Immunologic and Infectious Reactions in the Lung. Marcel Dekker Inc., New York, 1976, pp $101-130$.

9. Collier, A.M. \& Clyde Jr, W.A. Relationships between Mycoplasma pneumoniae and human respiratory epithelium. Infect Immunol 1971, 3: 694-701.

10. Biberfeld, $G$. Antibodies to brain and other tissues in cases of Mycoplasma pneumoniae infection. Clin Exp Immunol 1971, 8: 319-333.

11. Ponka, A. The occurrence and clinical picture of serologically verified Mycoplasma pneumoniae infections with emphasis on central nervous system, cardiac and joint manifestations. Ann Clin Res Suppl 1979, 24: 1-60. 